\title{
FUNCIONAMENTO INTESTINAL E A RELAÇÃO COM A INDEPENDÊNCIA FUNCIONAL DE INDIVÍDUOS COM LESÃO MEDULAR
}

\author{
BOWEL FUNCTION AND THE RELATIONSHIP WITH FUNCTIONAL INDEPENDENCE \\ OF INDIVIDUALS WITH SPINAL CORD INJURY \\ FUNCIONAMIENTO INTESTINAL Y RELACIÓN CON LA INDEPENDENCIA FUNCIONAL \\ DE INDIVIDUOS CON LESIÓN DE MÉDULA ESPINAL
}

Soraia Assad Nasbine Rabeh ${ }^{1}$, Paula Cristina Nogueira ${ }^{1}$, Maria Helena Larcher Caliri ${ }^{1}$

\begin{abstract}
RESUMO
Objetivos: Caracterizar indivíduos adultos que sofreram lesão de medula espinhal; avaliar a independência funcional considerando o nível da lesão; descrever a ocorrência de problemas de funcionamento intestinal e a relação com o nível de independência funcional. Método: Estudo observacional, transversal. Para investigar a independência funcional foi utilizada a escala Medida de Independência Funcional. Para avaliar o funcionamento intestinal, as orientações recebidas e as práticas de autocuidado, utilizaram-se as Diretrizes do Consórcio de Medicina da Lesão Medular. Resultados: Dos 22 participantes, 91\% eram homens, com média de idade de 38 anos (DP 11,7) e 50,0\% apresentavam dependência mínima e participavam de programa de reabilitação. Verificou-se alteração no padrão de funcionamento intestinal pós-lesão. Conclusão: A literatura enfatiza a importância de instituir programas de intervenção por meio de estratégias educativas para o controle do intestino neurogênico.
\end{abstract}

Descritores: Traumatismos da medula espinhal; Intestino neurogênico; Reabilitação; Atividades cotidianas.

\begin{abstract}
Objectives: To describe adults who have suffered spinal cord injury; evaluate their functional independence considering the level of injury; describe the occurrence of problems in bowel function and the relationship with the level of functional independence. Method: Cross-sectional and observational study. Functional independence was investigated using the Functional Independence Measure. To assess bowel function, the guidance the patients received and their self-care practices, we used the Guidelines of the Consortium for Spinal Cord Injury Medicine. Results: Of all 22 participants, 91\% were men, mean age 38 years (SD 11.7), and 50.0\% had minimal dependence and participated in a rehabilitation program. A change in the pattern of bowel function was observed after injury. Conclusion: Literature emphasizes the importance of establishing intervention programs through educational strategies for the control of neurogenic bowel.
\end{abstract}

Keywords: Spinal cord injuries; Neurogenic bowel; Rehabilitation; Activities of daily living.

RESUMEN

Objetivos: Caracterizar individuos adultos que sufrieron lesión medular espinal; evaluar su independencia funcional considerando nivel de la lesión; relatar ocurrencia de problemas del funcionamiento intestinal y la relación con el nivel de independencia funcional. Método: Estudio de obsenación, transversal. Para investigar la independencia funcional se utilizó la escala medida de independencia funcional. Para evaluar el funcionamiento intestinal, las orientaciones recibidas por el paciente y las prácticas de autocuidado, se usaron Directivas del Consorcio de Medicina de la Lesión Medular. Resultados: De 22 participantes, 91\% eran hombres, edad promedio 38 años (DE 11,7), 50,0\% presentaba dependencia mínima y participaba de programas de rehabilitación. Se verificó alteración en el patrón de funcionamiento intestinal post-lesión. Conclusión: La literatura enfatiza la importancia de instituir programas de intervención mediante estrategias educativas para controlar el intestino neurogénico.

Descriptores: Traumatismos de la médula espinal; Intestino neurogénico, Rehabilitación; Actividades cotidianas.

INTRODUÇÃO

A lesão da medula espinhal (LME) é considerada como um dos eventos incapacitantes mais graves e devastadores que pode atingir a pessoa, pois ocasiona a falência de uma série de funções vitais como locomoção, sensibilidade, sexualidade, alterações na função urinária e intestinal. , $^{1,2}$

As causas da LME podem ser de origem traumática e não traumática, sendo as de origem traumática as mais freqüentes, incluindo, os ferimentos por arma de fogo (FAF), acidentes automobilísticos, quedas e mergulho em águas rasas. ${ }^{1,2}$

A maior parte da população atingida pela LME traumática são indivíduos do sexo masculino e com menos de 40 anos. Observa-se, portanto, uma grande incapacidade que afeta uma população jovem e ativa, de forma repentina ocasionando repercussões psicobiológicas e psicossociais. ${ }^{2,3}$

Assim, o processo de reabilitação deve ser iniciado desde a fase aguda, nas unidades de terapia intensiva, para evitar complicações que podem ser catastróficas e onerosas. ${ }^{2}$

É necessário conhecer as características dos indivíduos com LME em termos de independência funcional, pois isso possibilita aos profissionais e aos centros de reabilitação estruturar-se para atenderem às demandas da população de forma mais efetiva e eficiente.

1. Departamento de Enfermagem Geral e Especializada da Escola de Enfermagem de Ribeirão Preto da Universidade de São Paulo (EERP-USP), Ribeirão Preto, SP, Brasil.

Trabalho realizado no Hospital das Clínicas da Faculdade de Medicina de Ribeirão Preto da Universidade de São Paulo, Ribeirão Preto, SP, Brasil.

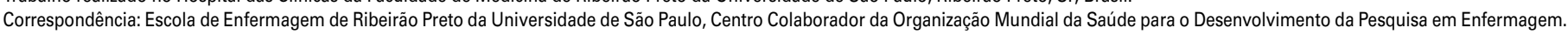
Av. dos Bandeirantes, 3900, Campus Universitário. 14040-902. Ribeirão Preto. SP. Brasil. soraia@eerp.usp.br 
Um instrumento muito utilizado para medir a independência funcional, chamado, Medida de Independência Funcional (MIF), é um instrumento de avaliação que foi desenvolvido para o acompanhamento de indivíduos em processo de reabilitação. Focaliza a atenção na efetiva realização de tarefas pelo indivíduo de maneira independente na rotina diária de avaliação. Foi traduzido para a língua portuguesa e validado para o uso ambulatorial e em pacientes subagudos e crônicos. ${ }^{4,5}$

A MIF engloba seis áreas de funcionamento: autocuidado, controle de esfíncteres, mobilidade, locomoção, comunicação e convivência social. Em cada área são avaliadas duas ou mais atividades perfazendo um total de 18 tarefas funcionais, 13 motoras e 5 cognitivas que são avaliadas em termos de independência da função, usando-se uma escala de sete pontos (1, referente a dependência total, e 7, a independência completa). Recomenda-se aplicá-la, no mínimo, em três momentos: o primeiro, no máximo até 72 horas da admissão; o segundo, dentro de 72 horas antes da alta e o terceiro, como seguimento do paciente após três a seis meses da alta do programa de reabilitação. ${ }^{4,5}$

A LME acarreta várias complicações incluindo o intestino neurogênico. Estudos enfatizam que a partir do nível neurológico da lesão, há expectativas de resultados para o funcionamento intestinal e medidas educativas devem ser implementadas. As complicações gastrintestinais, decorrentes do intestino neurogênico, podem ser classificadas em agudas ou crônicas, ou de acordo com o nível de comprometimento medular, cervical, torácica e lombar.,

O intestino reflexivo ou espástico resulta de lesão medular cervical ou torácica alta, em que ocorre a interrupção das mensagens entre cérebro e intestino, porém, a medula coordena os reflexos de eliminação fecal. Nesse caso, apesar de o paciente não sentir a necessidade de eliminar as fezes, a peristalse está presente e há resposta frente ao estímulo dígito-anal ou químico.

O intestino arreflexivo ou flácido origina-se da lesão lombar ou sacral, com diminuição da peristalse e do controle esfincteriano. Recomenda-se, para esses pacientes, a remoção manual das fezes, pois a resposta ao estímulo químico é muito incipiente. Na fase aguda da lesão cervical ou torácica alta podem ocorrer como complicações, sangramentos e íleo paralítico. Na fase crônica, a principal complicação é a impactação fecal, podendo modificar o estilo e a qualidade de vida.,7

O indivíduo com LME pode apresentar fases alternadas de constipação e incontinência fecal, antes de fazer o treinamento para a regulação intestinal, já que o componente muscular voluntário inibe o esvaziamento intestinal. ${ }^{6}$

Para atender às complicações intestinais de indivíduos com LME, foram construídas diretrizes com enfoque na prevenção de complicações, onde o enfermeiro expert tem papel preponderante na avaliação dos sinais e sintomas apresentados pelos pacientes e na proposição de plano de cuidados, envolvendo os familiares/ cuidadores na dinâmica da assistência. ${ }^{8}$

Acredita-se que muitos problemas poderiam ser evitados se houvesse maior integração e organização das atividades entre os profissionais envolvidos com este grupo de indivíduos. ${ }^{9}$

\section{OBJETIVOS}

Caracterizar indivíduos adultos que sofreram Lesão de Medula Espinhal (LME) entre janeiro de 2003 a Julho 2006; avaliar a independência funcional considerando o nível de lesão e descrever a ocorrência de problemas no funcionamento intestinal e a relação com o nível de independência funcional.

\section{MÉTODO}

Estudo seccional com abordagem quantitativa, aprovado pelo Comitê de Ética em Pesquisa (no processo 8902/2006). Primeiramente, foi realizado um levantamento em prontuários em instituições de saúde credenciadas ao Sistema Único de Saúde (SUS) em Ribeirão Preto para identificação dos sujeitos da pesquisa e posteriormente, foi realizada entrevistas no domicílio com indivíduos com LME. A população foi selecionada considerando os seguintes critérios de inclusão: indivíduos que sofreram LME no período de janeiro de 2003 a julho de 2006, atendidos em hospitais credenciados ao SUS e residentes no município de Ribeirão Preto, com idade igual ou superior a 18 anos capazes de responderem perguntas pertinentes ao estudo.

Para a avaliação da independência funcional foi utilizada a Escala para Medida de Independência Funcional - MIF. Este estudo limitou-se a avaliar a independência funcional após a alta.

Para a investigação da ocorrência das complicações intestinais foi utilizado um instrumento com a pergunta dicotômica (sim/não), tomando como referência o funcionamento intestinal antes e após a LME. Dentre as complicações, as variáveis foram impactação das fezes, hemorróidas, sangramento, incontinência fecal, constipação, diarréia e dor.

Os dados coletados foram digitados, organizados e armazenados em banco de dados Statistical Package of Social Sciences (SPSS) versão 11.6. Os resultados foram analisados utilizando testes estatísticos de Mann Whitney e o teste de correlação de Pearson, considerando a natureza das variáveis estudadas.

\section{RESULTADOS}

Foram entrevistados em domicílio 22 indivíduos que sofreram LME. Destes, 20 (91\%) eram do sexo masculino e 14 (63,6\%) tinham entre 20 e 39 anos (média 37,9 DP 11,6).

No momento da entrevista, a média de tempo pós-trauma era de 25 meses ( $d p=13$ meses) e a mediana de 24 meses. Quanto à etiologia do trauma, acidentes de motocicletas, quedas, colisão e acidentes automobilísticos foram as causas mais frequentes.

Onze indivíduos (50\%) sofreram lesão a nível cervical, seguida de lesão em nível torácico 10 (45,5\%) e um (4,5\%) em nível lombar.

Os valores da MIF total e seus domínios em relação ao nível da LME estão apresentados na Tabela 1.

As médias da MIF total dos sujeitos com LME em nível cervical e torácico apresentaram, no período até 12 meses pós-lesão, valores médios menores (média $=66,4 \mathrm{dp}=30$ ) em comparação com aqueles que tinham tempo pós-lesão acima de 13 meses ou mais (média =85,5 dp=23,9). Os valores médios da MIF motora mostraram aumento quando comparados aos desempenhos dos sujeitos nos períodos até 12 meses e 13 meses ou mais. Observou-se que o tempo de lesão constituiu fator relevante de influência sobre a MIF, entretanto, não foi verificada diferença estatisticamente significante entre os dois grupos de sujeitos.

Dos 22 pacientes estudados, nenhum apresentou grau de dependência completa, 11 (50\%) apresentaram dependência mínima, $6(27,3 \%)$ dependência máxima e $5(22,7 \%)$ independência moderada ou completa. Considerando os escores obtidos na avaliação pela MIF, 27,3\% tinham dependência máxima a moderada, sendo que a maior parte desses indivíduos tinha lesão no nível cervical e requeriam, portanto, assistência de até $50 \%$. No entanto, 7 pacientes

Tabela 1. Valores e variações da MIF total nos domínios motor e cognitivo, segundo o nível de lesão medular.

\begin{tabular}{c|c|c|c|c}
\hline Nível da lesão & $\begin{array}{c}\text { Valores e } \\
\text { variações }\end{array}$ & $\begin{array}{c}\text { MIF total } \\
\text { (escore 18-126) }\end{array}$ & $\begin{array}{c}\text { MIF motora } \\
\text { (escore 13-91) }\end{array}$ & $\begin{array}{c}\text { MIF cognitiva } \\
\text { (escore 5-35) }\end{array}$ \\
\hline Cervical $(n=11)$ & Média (dp) & $70,2(24,9)$ & $41,6(24,4)$ & $28,6(7,3)$ \\
\hline & Variação observ. & $25-101$ & $13-77$ & $12-35$ \\
\hline & Mediana & 62,0 & 28,0 & 32,0 \\
\hline Torácica $(n=10)$ & Média (dp) & $90,3(23,6)$ & $59,1(19,4)$ & $31,2(5,9)$ \\
\hline & Variação observ. & $48-112$ & $24-78$ & $15-35$ \\
\hline & Mediana & 98,0 & 65,5 & 32,5 \\
\hline Lombar $(n=1)$ & Média & 109,0 & 74,0 & 35,0 \\
\hline Total $(n=22)$ & Média (dp) & $81,1(25,9)$ & $51,0(23,4)$ & $30,0(6,6)$ \\
\hline & Variação observ. & $25-112$ & $13-78$ & $12-35$ \\
\hline & Mediana & 90,0 & 61,5 & 32,5 \\
\hline
\end{tabular}

(Ribeirão Preto, SP, 2007). 
(63,6\%), desse mesmo grupo, apresentaram grau de dependência mínima, necessitando de assistência de até 25\%.

Quanto ao funcionamento intestinal, houve alteração pós-lesão. Antes da lesão, a maioria dos pacientes $59,1 \%$, apresentava frequência de evacuação 1 vez ao dia, com características das fezes pastosas para $72,7 \%$ dos indivíduos e, após, houve mudança tanto na freqüência como na consistência, com maior número de indivíduos apresentando fezes de consistência dura. Quanto ao horário de evacuação, predominou o período da manhã, evidenciando manutenção do hábito.

Dos 22 pacientes, apenas $3(13,6 \%)$ tinham constipação antes da lesão e 15 (68,2\%) apresentaram constipação após a LME, observando-se, assim, interferência da condição no funcionamento intestinal.

Na Tabela 2 são apresentados os níveis de dependência para as atividades relacionadas ao funcionamento intestinal.

Observou-se que $8(36,4 \%)$ pacientes tinham dependência total para o uso do vaso sanitário em decorrência da dificuldade com o equipamento necessitando do cuidador para irem até o vaso sanitário. Entretanto, apenas 5 pacientes $(22,7 \%)$ tinham dependência para a dimensão controle de esfíncter-controle das fezes.

Os pacientes com independência modificada e independência completa tinham a mesma frequência $(22,7 \%)$ e a independência modificada para o uso do vaso sanitário foi encontrada em 7 pacientes $(31,8 \%)$

Quanto ao auxílio para o funcionamento intestinal, 17 (77,3\%) cuidadores prestaram assistência ao paciente, sendo que a estimulação mecânica com massagem abdominal e toque dígito-anal foram os cuidados mais frequentes, realizado por 9 cuidadores (41\%). Quanto ao nível de LME, onze indivíduos tinham lesão cervical, portanto, esperava-se que tivessem dependência para tal necessidade, por apresentarem intestino reflexivo. O controle nutricional foi referido por $16(72,7 \%)$ dos cuidadores como cuidado observado para o bom êxito no funcionamento intestinal.

Os pacientes com LME, em nível lombar, mostraram-se independentes para a maioria das necessidades, porém, para as atividades relacionadas à função intestinal, função vesical, cuidados pessoais/vestir-se, banho, mobilidade no leito, transferência cadeira de roda/banho, foram mais dependentes do que deveriam ser, se tivessem acesso à condições adequadas de reabilitação considerando as Diretrizes para a pratica clinica do Corsortium for Spinal Cord Medicine (CSCM). ${ }^{8}$

Tabela 2. Distribuição dos pacientes com lesão medular em relação ao nível de dependência dos cuidados para as atividades referentes ao funcionamento intestinal.

\begin{tabular}{c|c|c}
\hline Nível de dependência & $\begin{array}{c}\text { Controle de esfíncter: } \\
\text { controle das fezes }\end{array}$ & $\begin{array}{c}\text { Autocuidado: } \\
\text { uso do vaso sanitário }\end{array}$ \\
\hline & $\mathbf{f}(\%)$ & $\mathbf{f}(\%)$ \\
\hline 1 Dependência total & $5(22,7)$ & $8(36,4)$ \\
\hline 2 Dependência máxima & $0(0)$ & $0(0)$ \\
\hline 3 Dependência moderada & $2(9,1)$ & $0(0)$ \\
\hline 4 Dependência mínima & $2(9,1)$ & $2(9,1)$ \\
\hline $\begin{array}{c}\text { 5 Supervisão, estímulo } \\
\text { ou preparo }\end{array}$ & $3(13,6)$ & $2(9,1)$ \\
\hline 6 Independência modificada & $5(22,7)$ & $7(31,8)$ \\
\hline 7 Independência completa & $5(22,7)$ & $3(13,6)$ \\
\hline Total & $22(100,0)$ & $22(100,0)$ \\
\hline
\end{tabular}

Ribeirão Preto, SP, 2007.

\section{DISCUSSÃO}

As características sócio-demográficas dos indivíduos deste estudo corroboram resultados de outras pesquisas que encontraram uma população de adultos jovens, com idade entre 18 e 35 anos e na sua grande maioria do sexo masculino., ${ }^{3,6,10,11}$ Em relação às causas da LME, acidentes com veículo automotores e atos de violência têm sido observados no Brasil como frequente fator causal., $3,10,11$

Os resultados da MIF mostraram que os indivíduos com LME em nível cervical, apresentaram valores menores dos escores quando comparados com os indivíduos com LME em nível torácico e lombar, evidenciando que os primeiros apresentam menor independência funcional. Entretanto, a MIF cognitiva obteve o valor máximo de variação, indicando efeito teto independente do nível de lesão, resultado semelhante ao encontrado em outro estudo realizado com a mesma população na cidade de São Paulo. ${ }^{5}$

A literatura recomenda que é preciso investigar acerca do funcionamento intestinal antes do evento da LME, pois os hábitos anteriores referentes aos alimentos, líquidos ingeridos e atividades podem afetar o padrão de funcionamento intestinal após a lesão. As orientações devem ser direcionadas para o paciente e ao seu cuidador, considerando as características clínicas da LME, de modo a individualizar o cuidado e oferecer ferramentas para que possam desempenhar as ações que visam atender a necessidade intestinal com segurança. $6,7,12,13$

A predominância da LME para os indivíduos do estudo, em nível cervical e torácico alto, demonstra a necessidade de intervenção por meio de estratégias educativas para o tratamento do intestino neurogênico.

Segundo o Guia para Tratamento do Intestino Neurogênico a Adultos com Lesão de Medula Espinhal do Consortium for Spinal Cord Medicine (CSCM), ${ }^{8}$ traduzido para o português, ${ }^{6}$ deve ser instituído Programa de Treinamento para Controle da Função Intestinal para que os profissionais de saúde possam atuar junto aos pacientes e cuidadores, logo após a estabilização do choque medular, considerando o ambiente domiciliar e os recursos disponíveis.

Em estudo referente às dificuldades vivenciadas pelos pacientes com LME, após a alta hospitalar, foi identificado que os mesmos não receberam informações adequadas quanto às alterações possíveis e, tampouco quanto às práticas que visam minimizar ou prevenir as complicações inerentes à LME. Vários indivíduos referiram não ter recebido informações elementares durante a internação em hospital terciário. ${ }^{11}$

O tratamento da constipação intestinal em indivíduos com LME deve iniciar com a adoção de dieta equilibrada, rica em fibras e com ingestão adequada de líquidos, assim como aumento das atividades físicas diárias. ${ }^{6,13}$

Os indivíduos que são acometidos pela LME em nível cervical ou torácico, apresentam o intestino reflexivo ou espástico. Para esse padrão de intestino neurogênico, o paciente não sente a necessidade de eliminar as fezes, porém, existe a peristalse reflexa coordenada pela medula abaixo da lesão. O esfíncter anal permanece fechado, necessitando de estímulo digital, ou químico, para a eliminação das fezes, que devem ser macias para facilitar 0 esvaziamento intestinal. ${ }^{6}$ Nesse estudo, $50 \%$ dos indivíduos apresentaram LME em nível cervical e 45,5\% apresentaram a LME em nível torácico, portanto, necessitavam de orientações condizentes à sua condição clínica.

Os indivíduos com LME em nível lombar ou sacral apresentam o intestino arreflexivo ou flácido, com redução da peristalse e do controle reflexo do esfíncter anal. O intestino não é controlado pelos reflexos medulares, portanto, o paciente não sente necessidade de eliminar as fezes, podendo ocorrer eliminação de fezes espontânea. O cuidado intestinal não requer estimulantes químicos porque a resposta pode ser muito lenta, porém, as fezes devem ser removidas manualmente, por meio do toque dígito-retal até completo esvaziamento. ${ }^{6}$

Importante aspecto na educação do individuo para o cuidado intestinal é levá-lo a obter um padrão previsível de evacuação, que diminua a incontinência ou a impactação das fezes, assim como a interferência do funcionamento intestinal nas outras atividades da vida diária. Em estudos acerca da eliminação intestinal de indivíduos com $\mathrm{LME}$ em reabilitação, foram verificadas respostas frente a um programa de reeducação intestinal. A constipação foi a alteração mais freqüente. As pesquisas apontaram que o referido programa deveria abordar o treinamento para o esvaziamento intestinal, considerando cuidados quanto à padronização de horários para as evacuações, posicionamento, seja no vaso sanitário ou na cama, manipulação digital ao redor do ânus, incentivo à ingestão de líquidos e adequação da alimentação quanto ao tipo de alimento e horário para as refeições. $6,7,12,13$ 
Segundo as diretrizes para a prática clínica do $\mathrm{CSCM}^{8}$ em relação ao autocuidado intestinal, paciente com LME C1 a C5 são totalmente dependentes de um cuidador, C6 a C8 necessitam de assistência parcial de um cuidador, de T1 a T9 espera-se pela independência funcional, T10 a L1 e L2 a S5 também são independentes no cuidado intestinal em condições adequadas. ${ }^{8}$ Assim, o exame neurológico deve ser realizado e compartilhado entre os multiprofissionais a fim de se estabelecer metas a serem alcançadas no processo de reabilitação.

A reabilitação deve se iniciar desde a primeira hospitalização, após a LME, e atender às necessidades específicas de cada indivíduo, com abordagem interdisciplinar que pode prevenir as complicações iniciais e diminuir o custo total da assistência. Atraso para iniciar essas ações pode influenciar negativamente a sua independência funcional e prolongar o tempo de reabilitação. 2,14-16

\section{CONCLUSÕES}

Quanto ao funcionamento intestinal, verificou-se que os indivíduos, em sua maioria, demonstraram dependência para a necessidade intestinal e necessitavam de auxílio do cuidador principalmente para o uso do vaso sanitário, sugerindo que a falta de equipamentos apropriados como, por exemplo, cadeira de rodas de fácil manejo poderia constituir limitação para o melhor o nível de independência.

As barreiras para a obtenção do cuidado adequado e do material necessário precisam ser identificadas para minimizar a ocorrência de complicações. O cuidado de qualidade deve ser esperado como norma em cada instituição de saúde. O CSCM mostra, através das diretrizes, que há expectativa de resultados funcionais em relação ao nível da LME, porém, enfatiza o recurso do equipamento para a obtenção de tais resultados.

Quanto ao autocuidado intestinal, existem graus de independência funcional associados a vários fatores tais como nível da LME, tipo de lesão, comportamento do indivíduo e motivação da família. Os programas de treinamento do funcionamento intestinal devem considerar os padrões anteriores à lesão para estabelecer metas de reabilitação. Tais programas devem ser iniciados tão logo se estabilize o quadro de choque medular e incluir o preparo do paciente, cuidador e profissionais envolvidos com a assistência.

Embora a independência funcional esperada, ao final do primeiro ano após o evento LME, nem sempre pode ser alcançada, devido às variações referentes à idade, sexo, condições clínicas e comorbidades, a assistência prestada nos primeiros meses, após a lesão é fundamental para monitorar, tratar e prevenir as complicações que levam a futura morbidade e mortalidade.

Observa-se, na prática profissional, que a assistência à saúde, de um modo geral, está estruturada de forma a mostrar lacunas quanto ao aspecto do preparo para a continuidade do cuidado, no domicílio, após a alta hospitalar.

Apesar da complexidade que envolve os pacientes com LME, acredita-se na importância e necessidade de modificar a prática de enfermagem, a forma de assistir o individuo e a família, visando melhorar a qualidade dessa assistência e atender às necessidades do individuo e família/cuidador, para que estejam em condições de enfrentar os desafios do cotidiano.

Compreende-se, a partir dos resultados desta pesquisa, que a intervenção de enfermagem deve avançar além dos limites da prática ritualística e rotineira, de modo a construir um modelo de assistência que privilegie a necessidade real do cliente, enquanto agente participante do seu processo de cuidado. Para tal, o enfermeiro deve dispor de atributos legais e éticos da Sistematização da Assistência de Enfermagem, de modo a individualizar o cuidado e alcançar melhores resultados para atendimento dessa população, em parceria com os outros membros da equipe multidisciplinar.

Todos os autores declaram não haver nenhum potencial conflito de interesses referente a este artigo.

\section{REFERÊNCIAS}

1. Greve JMDA, Casalis MEP, Barros Filho TEP. Diagnóstico e tratamento da lesão da medula espinhal. São Paulo: Roca; 2001.

2. Ares MJJ, Cristante ARL. Lesão medular. In: AACD- Medicina e Reabilitação. São Paulo: Artes Médicas; 2007.

3. Nogueira PC, Caliri MHL, Haas VJ. Profile of patients with spinal cord injuries and occurrence of pressure ulcer at a university hospital. Rev Latino Am Enferm. 2006; 14(3):372-7.

4. Riberto M, Miyazaki MH, Jorge Filho D, Sakamoto H, Battistella LR. Reprodutibilidade da versão brasileira da medida de independência funcional. Acta Fisiátrica. 2001:8(1):45-52.

5. Riberto M, Miyazaki MH, Jucá SSH, Sakamoto H, Pinto PPN, Battistella LR. Validação da versão brasileira da medida de independência funcional. Acta Fisiátrica. 2004;11 (2):72-6.

6. Caliri MHL, Furlan MLS, Defino HL. Management of neurogenic bowel in adults with spine cord injury. Guidelines for an evidence-based practice. Coluna/Columna. 2005;4(2):102-5.

7. Krassioukov A, Eng JJ, Claxton G, Sakakibara BM, Shum S. Neurogenic bowel management after spinal cord injury: A systematic review of the evidence. Spinal Cord. 2010;48(10):718-33.

8. Paralyzed Veterans of America. Outcomes following traumatic spinal cord injury: a clinical practice guideline for health care professionals. Washington (DC): Paralyzed Veterans of America; 1999.

9. Rabeh SAN, Caliri MHL. Functional ability in individuals with spinal cord injury. Acta Paul Enferm. 2010;23(3):321-7.
10. Vasconcelos ECLM, Riberto M. Clinical characterization and description cases of vertebral spinal fracture in the municipality of Ribeirão Preto, proposals for a spinal cord trauma prevention program. Coluna/Columna. 2011;10(1):40-3.

11. Nogueira PC. Sobrecarga do cuidado e qualidade de vida relacionada à saúde de cuidadores de indivíduos com lesão medular [tese]. Ribeirão Preto (SP): Universidade de São Paulo - Escola de Enfermagem de Ribeirão Preto; 2010.

12. Coggrave M, Norton C, Wilson-Barnett J. Management of neurogenic bowel dysfunction in the community after spinal cord injury: a postal survey in the United Kingdom. Spinal Cord. 2009;47(4):323-30.

13. Bruni DS, Strazzieri KC, Gumieiro MN, Giovanazzi R, Sá VG, Faro ACM. Physiopathological aspects and nursing care on rehabilitation of patient with spinal cord injury. Rev Esc Enferm USP. 2004;38(1):71-9

14. Riberto M, Pinto PPN, Sakamoto H, Battistella LR. Independência funcional de pacientes com lesão medular. Acta Fisiátrica. 2005;12(2):61-6.

15. Kirshblum SC, Priebe MM, Ho CH, Scelza WM, Chiodo AE, Wuermser LA. Spinal cord injury medicine. 3. Rehabilitation phase after acute spinal cord injury. Arch Phys Med Rehabil. 2007; 88(3 Suppl 1):S62-70.

16. Custódio NRO, Carneiro MR, Feres CC, Lima GHS, Jubé MRR, Watanabe LE, Saliba LGRSO, Daher S, Garcia ACF. Lesão medular no Centro de Reabilitação e Readaptação Dr. Henrique Santillo (CRER- GO). Coluna/Columna. 2009;8(3):265-8. 